\title{
Análisis edad-periodo-cohorte de la mortalidad por accidentes de tráfico en España
}

\author{
Carmen Saiz-Sánchez, ${ }^{(1,2)}$ D aniel Bautista-Rentero, ${ }^{(1)}$ D olores Corella-Piquer, ${ }^{(2)}$ \\ Silvia Cortina-Birlanga, ${ }^{(1,2)}$ José Ignacio González-A rraez ${ }^{(2)}$
}

\begin{abstract}
Saiz-Sánchez C, Bautista-Rentero D, Corella-Piquer D, Cortina-Birlanga S, González-Arraez JI. A nálisis edad-periodo-cohorte de la mortalidad por accidentes de tráfico en España.

Salud Publica Mex 1999;41:170-176.
\end{abstract}

\section{Resumen}

Objetivo. Estudiar la evolución de la mortalidad por accidentes de tráfico en España y su posible aplicación a un modelo edad-periodo-cohorte, así como el efecto que pueden tener algunas medidas de seguridad vial seleccionadas. Material y métodos. Se obtuvieron las tasas de mortalidad por accidentes de tráfico y las tasas en intervalos quinquenales de edad para cada sexo, lo que permite su estudio como tasas específicas de edad por cohortes de nacimiento. Para determinar la asociación entre las medidas de seguridad vial seleccionadas y la mortalidad se han construido modelos de regresión de Poisson. Resultados Se observaron dos ondas evolutivas en la mortalidad por accidentes de tráfico. Respecto a la edad, no podemos hablar de un efecto claro; tampoco se encontró un efecto cohorte ni para varones ni para mujeres. En relación con las medidas de seguridad vial, se discutió la consistencia que guardaban los modelos seleccionados con los resultados gráficos, y se obtuvo que el uso obligatorio del casco y de las luces de cruce en motocicletas se ha asociado significativamente a la reducción de la mortalidad (RR $0.73, p<0.05$ ). Conclusiones La mortalidad por accidentes de tráfico en España muestra un ligero incremento en el periodo estudiado. Este comportamiento de las tasas no puede ser explicado suficientemente por los efectos de la edad, el perio do de diagnóstico de la muer te y la cohor te de nacimiento; en cambio las medidas de seguridad vial introducidas se valoraron positivamente.

Palabras clave: modelos edad-periodo-cohorte; accidentes de tránsito; mortalidad; factores de riesgo; España

\author{
Saiz-Sánchez C, Bautista-Rentero D, Corella-Piquer D, \\ Cortina-Birlanga S, González-Arraez JI. \\ Age-period-cohort analysis \\ of traffic accident \\ mortality in Spain. \\ Salud Publica Mex 1999;41:170-176.
}

\begin{abstract}
A bstract
Objective.To study the evolution of traffic accidents mortality in Spain and its possible application to an age-periodcohort analysis, as well as the effect of selected road safety measures. Material and methods Road accidents rates of mortality were obtained, and five-year interval age rates for each sex, which allows the study of specific rates of age by birth cohorts. To determine the association between the selected road safety measures and mortality, Poisson regression models were adjusted. Results. Two waves emerge in the evolution of traffic accidents. There was no clear effect with respect to age, nor was there a cohort effect for men or women.As to the road safety measures, we discuss the consistency between the selected models and graphic results. The compulsory use of helmet and of crossing lights is significantly asso ciated to a reduction in mortality ( $R R$ R 0.73 , $p<0.05)$. Conclusions Road accidents mortality shows a slight increase in the studies period. This rate performance cannot be sufficiently explained by age effects, diagnostic period nor birth cohort, how ever, road safety measures are considered positive.
\end{abstract}

Key words: age-period-cohort models; road accidents;mortality; risk factors; Spain

(1) Unidad Mixta de Investigación, Hospital Clínico Universitario, Universidad de Valencia, España.

(2) Departamento de Medicina Preventiva y Salud Publica, Facultad de Medicina, Universidad de Valencia, España.

Fecha de recibido: 20 de marzo de 1998 - Fecha de aprobado: 11 de marzo de 1999

Solicitud de sobretiros: C armen Saiz Sánchez. D epartamento de Medicina Preventiva y Salud Pública. Facultad de Medicina. A venida Blasco Ibáñez 17, 46010 Valencia, España. 
$E$ n la actualidad el tráfico se ha convertido en una de las fuentes de riesgo para la salud más importantes en los llamados países desarrollados, además de que constituye una pesada carga en lo que respecta a la pérdida de vidas humanas. ${ }^{1-3}$ La importancia sanitaria de los accidentes de tráfico reside, fundamentalmente, en tres pilares de estudio: la alta morbilidad y mortalidad que producen, las secuelas físicas y psíquicas que generan, y el elevado costo económico que representan. ${ }^{4}$

En España, según datos de la Encuesta Nacional de Salud llevada a cabo durante el año $1995,{ }^{5} 1.6 \%$ de la población se ha visto implicada en algún tipo de accidente de tráfico. Además, en ese país se dispone de información muy completa acerca de las defunciones por accidentes de tráfico, a partir de dos fuentes de datos: los boletines informativos de la Dirección General de Tráfico (DGT) y los Movimientos naturales de la poblacion española publicados por el Instituto Nacional de Estadística (INE) ${ }^{6,7}$ De acuerdo con estas fuentes, lamentablemente los indicadores de siniestralidad por accidentes de tráfico en España son altos en comparación con los de otros países del entorno, ${ }^{8-10}$ por lo que pasan a formar parte de una de las estadísticas de mortalidad más preocupantes de Europa. ${ }^{10}$

Teniendo en cuenta esta situación, se planteó la necesidad de llevar a cabo un estudio para conocer con mayor profundidad la epidemiología de los accidentes de tráfico en España. Sin embargo, se entiende que una investigación de los accidentes tendente a aclarar la naturaleza de sus agentes etiológicos, como la fatiga de los conductores, el consumo de drogas en general, la antigüedad y la conservación de los vehículos u otros factores, se aleja del objetivo inicial, además de que algunos de esos agentes ya han sido abordados en publicaciones específicas. ${ }^{4,11-14}$ Por tal motivo, en este trabajo se plantea, en primer lugar, el estudio de la evolución de la mortalidad producida por los accidentes de tráfico en España entre 1970 y 1993 en la población total y según género, desglosando de manera descriptiva los llamados efectos edad (cambio debido a factores biológicos), periodo (cambio en la frecuencia de la enfermedad, específico del periodo de observación) y cohorte (cambio en la frecuencia que es compartido por todos los miembros de la población, seguida a partir de un tiempo común). En segundo lugar, se busca conocer si la evolución de la mortalidad a lo largo del periodo estudiado responde, de alguna manera, a las medidas de seguridad vial (como, por ejemplo, la obligatoriedad del uso de cinturón de seguridad, el establecimiento del límite máximo de velocidad, etc.) que a lo largo de esos años se han ido implantando en España.

\section{Material y métodos}

Las fuentes de datos de las defunciones por accidentes de tráfico para el periodo 1970 a 1993 en España han sido los Movimientos naturales de la población española que publica el INE. ${ }^{7}$ Para dicho periodo se han consultado las revisiones 7a., 8a. y 9a. de la Clasificación Internacional de Enfermedades (CIE), en especial las nomenclaturas correspondientes a "Accidentes de tráfico de automóviles" (E810-E825), de la 7a. revisión, y "Accidentes de tráfico de vehículos de motor" (E810E819), de las revisiones 8a. y 9a. ${ }^{7}$

También se han consultado los Boletines Informativos de Accidentes publicados por la DGT, con el fin de conocer la entrada en vigor de las principales medidas de seguridad vial que podrían explicar los cambios en la evolución de la mortalidad por esta causa de defunción. ${ }^{8}$ Aunque en sus boletines la DGT también informa periódicamente acerca de las defunciones, se optó por utilizar la correspondiente al INE que considera "muerto por accidente de tráfico" a aquel que fallece hasta un año después del accidente y como consecuencia de él; por este motivo, el periodo de 24 horas que contabiliza la DGT (es decir, define muerte por accidente de tráfico a aquella que sucede dentro de las 24 horas siguientes al mismo) nos obligaría multiplicar, si utilizáramos estas defunciones, por un factor corrector, que se estima en aproximadamente 1.3 en algunos estudios. ${ }^{15}$ Asimismo, el criterio del INE también permite establecer comparaciones con otros países, ya que los agrupa según la CIE. ${ }^{16}$ Para la obtención de los datos demográficos se han consultado los Censos de Población y las Proyecciones de la población española para los años intercensales (INE). ${ }^{17,18}$

El estudio de la evolución de la mortalidad por accidentes de tráfico y de los efectos edad, periodo y cohorte de nacimiento, se ha llevado a cabo mediante métodos gráficos con escala normal o semilogarítmica; este aspecto se ha abordado únicamente de manera descriptiva. En primer lugar, se calcularon las tasas de mortalidad específicas por grupos de edad y sexo, expresándolas por cada 100000 personas-año. Los seis grandes grupos de edad considerados para el estudio fueron: 0-14, 15-29, 30-44, 45-49, 60-74, y el grupo de 75 y más años. Posteriormente, para tener una medida resumen y poder comparar la evolución de la mortalidad con el tiempo, se calcularon anualmente, desde 1970 hasta 1993, las tasas mortalidad por accidentes de tráfico ajustadas por edad y sexo mediante el llamado método directo; esta medida de frecuencia se expresó en defunciones por cada 100000 personasaño. La población estándar empleada fue la europea propuesta por la Organización Mundial de la Salud 
(OMS). ${ }^{19}$ El ajuste de líneas de tendencia de la mortalidad para todo el periodo se llevó a cabo mediante métodos de regresión lineal simple; como variable dependiente se consideraron las tasas ajustadas de defunción, y como independiente, el año de las mismas. Como paso previo para aplicar este modelo se calculó el estadístico de Durbin-Watson, y se obtuvieron valores de 1.2 y 1.3 para hombres y mujeres, respectivamente. Aunque son valores elevados, esta correlación serial de los residuales permite la aplicación de un modelo de regresión lineal.

Para el análisis de mortalidad por cohortes de nacimiento, se calcularon tasas específicas de mortalidad por accidentes de tráfico, por grupos quinquenales de edad y sexo, y se tabularon frente al tiempo (intervalos quinquenales). Se trazaron las diagonales de forma paralela en la tabla; las líneas de dicha ordenación representan las cohortes de interés. ${ }^{19-20}$ Posteriormente, al calcular retrospectivamente los intervalos quinquenales de nacimiento de cada una de las cohortes, se representaron las tasas específicas de mortalidad frente al promedio de edad de defunción en cada uno de los grupos.

Por último, se ha llevado a cabo una análisis de regresión de Poisson con términos autorregresivos ${ }^{21}$ para estudiar, desde una perspectiva ecológica, la posible asociación entre la mortalidad por accidentes de tráfico en España durante el periodo considerado y la implantación de importantes medidas de seguridad vial en este país. Se empleó la regresión de Poisson, ya que la mortalidad por accidentes de tráfico seguía dicha distribución. Para controlar la posible correlación temporal al analizar los datos longitudinales de la serie, en el modelo de regresión se probó la significación estadística de los distintos retardos de la mortalidad $(1,2,3 \ldots)$ manteniéndolos en el modelo si alcanzaban un valor de $p<0.05$. Las medidas de seguridad vial seleccionadas fueron: la introducción de un límite máximo de $0.8 \mathrm{~g}$ de alcohol en sangre de los conductores (1973), el uso obligatorio del cinturón de seguridad en carretera (1974), límites de velocidad en vías urbanas e interurbanas (1975) y obligatoriedad del uso de casco y luz de cruce en motocicletas (1980). Estas medidas de seguridad vial se introdujeron en el modelo de regresión de Poisson como variables independientes indicadoras, y fueron codificadas como $1 \mathrm{a}$ partir del año siguiente de su implantación y como 0 para el resto de años. El análisis estadístico se llevó a cabo con el paquete estadístico SPSS para Windows, complementado con el programa EGRET para las regresiones de Poisson.

\section{Resultados}

\section{Evolución de la mortalidad a causa de los accidentes de tráfico}

En la figura 1 se presenta la evolución de las tasas de mortalidad por accidentes de tráfico ajustadas por edad desde 1970 hasta 1993 en España. La mortalidad así vista fue ligeramente creciente en ambos sexos, si bien no hubo una tendencia lineal estadísticamente significativa $(p<0.05)$. A lo largo del periodo se puede observar claramente la existencia de dos ondas evolutivas en ambos sexos, aunque más acentuadas para los hombres; la primera abarca de 1974 a 1981, y la segunda desde 1982 hasta 1993. En la población masculina el valor máximo de la primera y la segunda onda, en tasas por $10^{5}$ hombres-año, se alcanzó en 1978 y 1989, con 27.64 y 32.16, respectivamente. Esta última es la tasa de mortalidad más alta alcanzada en el estudio.

La mortalidad en mujeres también hace evidente las dos ondas evolutivas, y es muy similar a la evolución observada en los hombres, aunque la mortalidad de forma genérica es triplicada o cuadruplicada por la población masculina en el periodo de estudio.

\section{Efectos de la edad, el periodo y la cohorte}

La figura 2 recoge la evolución de las tasas específicas anuales de mortalidad por accidentes de tráfico en hombres por los distintos grupos de edad estructura-

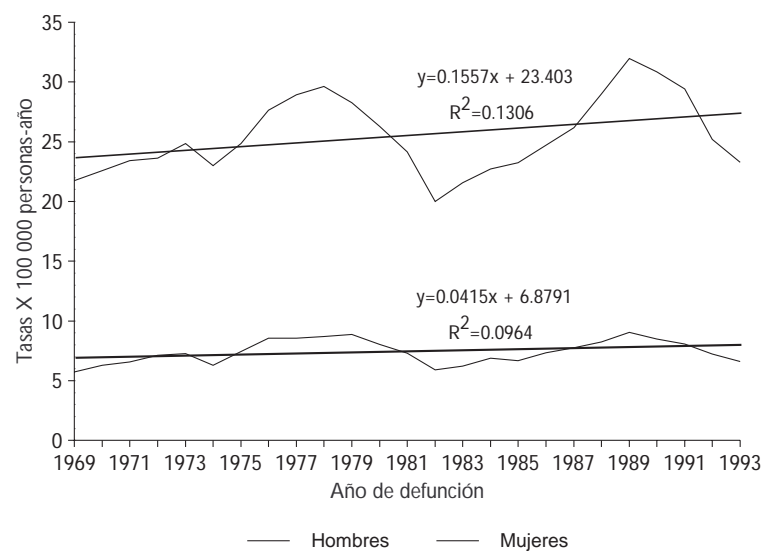

FIGURA 1.TASAS DE MORTALIDAD POR ACCIDENTES DE TRÁFICO AJUSTADAS POR EDAD. ESPAÑA, 1970-1993 


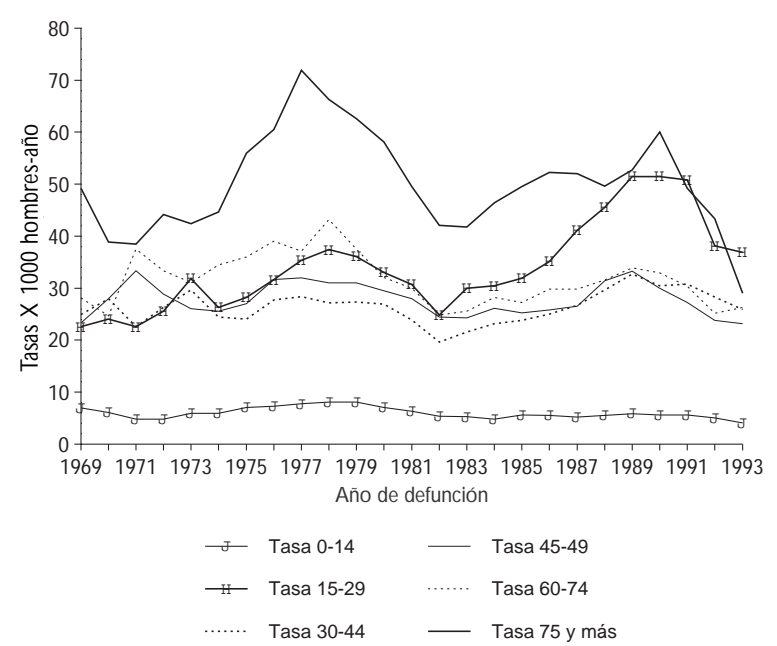

FIGURA 2.T ASAS DE MORTALIDAD POR ACCIDENTES DE TRÁFICO ESPECÍFICAS Y POR GRUPOS DE EDAD EN POBLACIÓN MASCULINA. ESPAÑA, 1970-1993

dos para el estudio. Sólo se incluye la representación gráfica de la tendencia en la población masculina, ya que en los dos sexos las evoluciones temporales son semejantes. En ambos sexos, el grupo de sujetos mayores de 75 años destaca claramente, seguido por el de 60-74 en mujeres. Por el contrario, en hombres, el segundo lugar por edad lo ocupan los grupos de 60-74 años, de 1970 a 1980. A partir de 1982, el segundo lugar lo ocupa el grupo de 15-29 años. Se observa con ello que la mortalidad en los jóvenes se incrementa con el paso del tiempo, mientras que para los mayores de 60-74 años, tiende a descender.

Para efectuar una simplificación en la visualización de los resultados por edad, se estudió la distribución por edad y evolución de las tasas específicas por accidentes de tráfico en hombres y mujeres durante los años 1970, 1980, y 1990 como resumen de las tres décadas que abarca el periodo (resultados no mostrados). De nuevo se observa una distribución bimodal (15-29 años y mayores de 75 años) en ambos géneros, más patente entre los hombres. A lo largo de las tres décadas, las personas jóvenes tienen cada vez una mayor mortalidad, y ésta desciende en los de mayor edad, por lo que no se puede hablar de un efecto edad.

Para estudiar el fenómeno cohorte, en las figuras 3 y 4 se representan las tasas de mortalidad por grupos de edad en función de la cohorte de nacimiento. En la primera cohorte se ha considerado a los hombres y mujeres nacidos entre 1914 y 1918, mientras que la última cohorte representada corresponde a los nacidos entre 1964 y 1968. En los hombres (figura 3), al

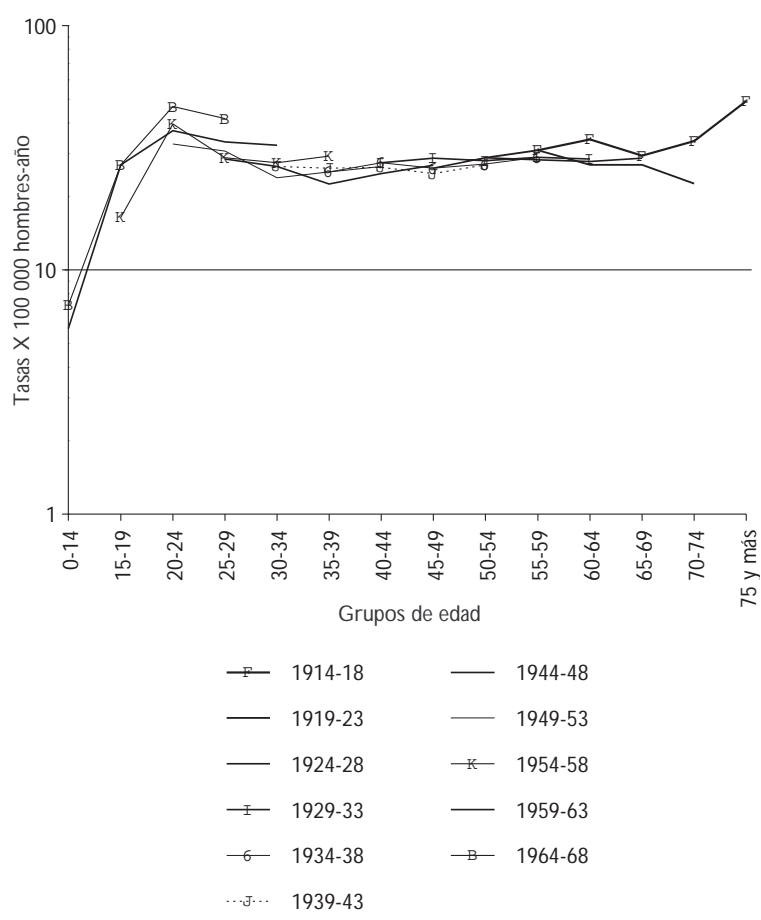

Figura 3.TASAS DE MORTALIDAD POR ACCIDENTES DE TRÁFICO SEGÚN COHORTES DE NACIMIENTO EN POBLACIÓN MASCULINA. ESPAÑA, 1970-1993

analizar las tasas por cohortes de nacimiento, no se observa un claro efecto edad-cohorte, ya que las líneas que representan las distintas cohortes no evidencian paralelismo; más bien se produce un avance entrecruzado que indica un ligero efecto periodo. El efecto cohorte habría mostrado que cada cohorte de nacimiento presenta mayores tasas de mortalidad que las precedentes. En las mujeres (figura 4), si bien podría hablarse de un ligero efecto cohorte en las generaciones más jóvenes, en el cómputo global tampoco se hace evidente este efecto.

\section{Efecto de las medidas de seguridad vial}

Para estudiar el efecto de las medidas de seguridad vial en la mortalidad por accidentes de tráfico en España, a lo largo del periodo seleccionado, se llevó a cabo una regresión de Poisson con términos autorregresivos, según los criterios descritos con anterioridad. Debido a la evolución similar de la mortalidad en hombres y mujeres, se han considerado las defunciones para ambos géneros conjuntamente en un mismo modelo de regresión. En el cuadro I se presentan los coeficientes de regresión, el error típico de la estimación y la 


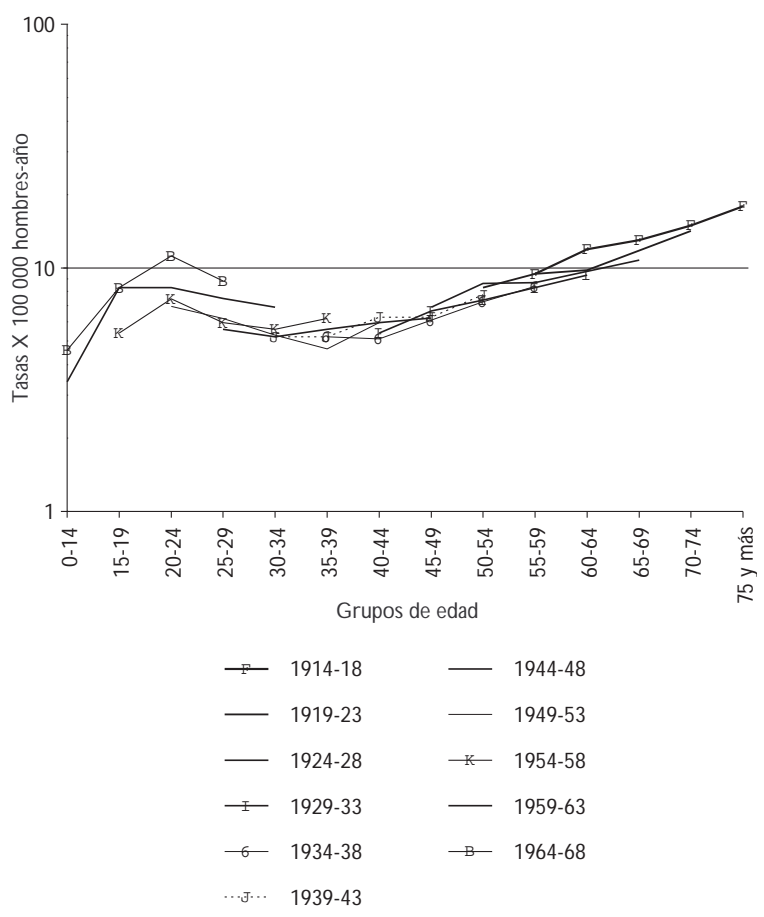

FigURA 4.T ASAS DE MORTALIDAD POR ACCIDENTES DE TRÁFICO SEGÚN COHORTES DE NACIMIENTO EN POBLACIÓN FEMENINA. ESPAÑA, 1970-1993

Cuadro I

Asociación ENTRE ALGUNAS MEDIDAS DE SEGURIDAD VIAL Y LA MORTALIDAD POR ACCIDENTES DE TRÁFICO. Regresión de Poisson. España, 1970-1993

\begin{tabular}{|c|c|c|c|}
\hline Variables en el modelo* & $\begin{array}{l}\text { oeficiente }(\beta) \\
\text { de regresión }\end{array}$ & $\begin{array}{l}\text { Error } \\
\text { típico }\end{array}$ & $\begin{array}{l}\text { Signifi- } \\
\text { cación (p) }\end{array}$ \\
\hline Año de defunción (tendencia) & 0.026 & 0.015 & $0.09 n$ \\
\hline Consumo de alcohol & -0.099 & 0.127 & 0.442 \\
\hline Uso obligatorio cinturón seguridad & 0.069 & 0.151 & 0.648 \\
\hline Límite máximo de velocidad & 0.067 & 0.148 & 0.687 \\
\hline Uso obligatorio del casco y luz de cruce & -0.307 & 0.117 & 0.017 \\
\hline
\end{tabular}

significación estadística de las variables independientes probadas en el modelo, en el que además se incluyen los términos autorregresivos de la mortalidad que resultaron estadísticamente significativos (retar- dos 1 y 2), para controlar la influencia de una posible autocorrelación temporal en la serie. De todos las medidas de seguridad vial analizadas, sólo la correspondiente a la obligatoriedad del uso de casco y de luz de cruce en motocicletas resultó asociada de manera negativa ( $\beta=0.307$ ) y estadísticamente significativa $(p=0.017)$ con la mortalidad por accidentes de tráfico en ambos géneros. El riesgo relativo (RR) correspondiente a esta medida se calculó a partir de su coeficiente de regresión, y se obtuvo un valor de 0.74 . La introducción de una cifra máxima de alcoholemia en la sangre de los conductores también se asoció negativamente con la mortalidad, aunque sin alcanzar la significación estadística.

\section{Discusión}

A partir de los resultados obtenidos en el presente estudio sobre la mortalidad por accidentes de tráfico en España, se ha observado una tendencia ascendente y preocupante, apenas modificada por las medidas de seguridad vial introducidas. Aunque la mortalidad por accidentes de tráfico se considera como una causa de mortalidad evitable, ${ }^{22}$ es difícilmente prevenible y compleja de estudiar, y rompe con patrones como los hoy aplicados de edad-periodo-cohorte, afectando mucho más al hombre que a la mujer; no obstante, cabe aclarar que los jóvenes (15-29 años) constituyen el grupo con mayor riesgo de sufrir una lesión fatal por accidente de tráfico en los últimos años. Esta valoración lleva a plantear el diseño y el mejoramiento de los programas de prevención en este grupo de edad. Con especial énfasis, también debería considerarse a los mayores de 60 años, ya que distintos estudios ${ }^{1,23}$ ponen de manifiesto que las habilidades necesarias para una conducción segura empiezan a deteriorarse a la edad de 55 años, y disminuyen de forma importante después de los 75 años. ${ }^{24,25}$

A lo anterior es necesario agregar que sólo en 1995, según datos proporcionados por la DGT, se incorporaron 577000 nuevos conductores que superan ya los 17 millones de conductores que viajan por las carreteras españolas y de los cuales 13\% están entre los 18-24 años; $53 \%$, entre los 25-44; $27 \%$, entre los $45-64$, y $6 \%$ tiene más de 65 años. Tomando en cuenta que este último grupo de edad irá en crecimiento de acuerdo con la evolución demográfica, los programas de prevención de accidentes de tráfico deberán contemplar medidas específicas a fin de reducir el riesgo en los grupos de edad más avanzados. ${ }^{26}$

Respecto a la edad, la bimodalidad encontrada, esto es, la aparición de dos picos separados en las cur- 
vas de mortalidad, es siempre interesante, ya que indica que la mortalidad no es homogénea y que podría ser apropiadamente dividida en dos, lo que sugeriría la existencia de diferencias causales. ${ }^{27}$ Asimismo, la incorporación de la mujer a la obtención del permiso para conducir en un $34 \%$ explica la menor mortalidad en este grupo, la cual, sin embargo, puede ir incrementándose en un futuro próximo.

Otras circunstancias como la importancia del urbanismo creciente, el aumento de la potencia de los vehículos, y el incremento y la complejidad de los bienes de consumo son, sin lugar a duda, condiciones que aumentan las situaciones de riesgo potencial frente a importantes beneficios y cuya valoración ligada a los accidentes de tráfico incluso es difícil; aunque ya seamos capaces de recoger las estadísticas, aún es complejo encontrar modelos como el aplicado de edadperiodo-cohorte, que puede explicar su comportamiento, o como el de regresión múltiple de Poisson en el que se puede encontrar la importancia explicativa de algunos factores considerados, como ha sido el uso obligatorio del casco y luz de cruce en motocicletas. ${ }^{26,28-29}$

A partir de 1960 la DGT ha llevado a cabo campañas de prevención que tratan de ser efectivas; ;6,30-32 para ello se han desarrollado programas educativos a distintos niveles -utilizando incluso los medios de comunicación- y se han incrementado las sanciones de tal modo que se puede retirar el carnet de conducir; además, se han extendido los medios de control de alcoholemias, y existen radares móviles para detectar la velocidad de los vehículos. Muchas de estas variables, que se consideraron independientes y que se ha pretendido relacionarlas con la mortalidad en el mismo periodo, requieren de una secuencia temporal adecuada que permita establecer las posibles causas-efecto. Por dicha razón, es necesario orientarse hacia los estudios que proporcionen mayores intervalos de tiempo y posibles explicaciones acerca de un impacto sobre la mortalidad, para evaluar cuáles medidas de prevención están dando mejores resultados y cuáles se deben incrementar y mejorar. ${ }^{28}$

De las medidas de seguridad vial seleccionadas, la que parece relacionarse de forma más evidente, al menos desde el punto de vista estadístico, es el uso obligatorio del casco y de la luz de cruce en motocicletas introducida a partir de 1980; ello supuso un descenso de la mortalidad por accidentes de tráfico en ambos géneros. Si bien el uso del casco sólo tiene impacto sobre las defunciones por accidentes donde están involucrados motociclistas, la obligatoriedad del uso de las luces de cruce en motocicletas también reduce la mortalidad por accidentes entre los ocupantes de otro tipo de vehículos, lo que contribuye a un descenso significativo global de la mortalidad. Por tanto, se considera que sería deseable fomentar las campañas informativas con el fin de lograr un mayor cumplimiento de estas y otras medidas, así como extender la obligatoriedad de uso del casco a los ciclistas.

Cabe señalar que el resto de medidas de seguridad vial analizadas no han mostrado una asociación estadística con la mortalidad por accidentes de tráfico en el presente estudio. Por supuesto que ello no significa que no ejerzan su indudable efecto protector, sino que, probablemente, dicho efecto está enmascarado por otro conjunto de factores (de seguridad vial, socioeconómicos, humanos, infraestructuras, etc.) no considerados en este trabajo, o bien, que los indicadores de mortalidad utilizados no son los más apropiados para evidenciar su influencia. ${ }^{26,28,30-32}$

Por último, cabe comentar que el estudio edadperiodo-cohorte también podría haberse llevado a cabo mediante un modelo matemático tal que:

$$
\log (\operatorname{tas} a)_{i}=a+\beta_{1 i} E+\beta_{2 i} P+\beta_{3 i} C+e_{i}
$$

donde

$\mathrm{a}=$ el logaritmo de la tasa de incidencia en el estrato de población considerado como referencia,

$\beta_{1 \mathrm{i}} \beta_{2 \mathrm{i}^{\prime}} \beta_{3 \mathrm{i}}=$ los logaritmos de los riesgos relativos de los diferentes grupos de edad, periodo y cohorte, respectivamente, en relación con la mortalidad en el grupo de referencia.

Este modelo asume que las tasas siguen una distribución de Poisson, y los estimadores de los coeficientes de regresión se obtienen por el método de máxima verosimilitud. ${ }^{33}$ Sin embargo, el empleo de estos modelos matemáticos, además de su complejidad, plantea problemas de interpretación de los coeficientes obtenidos, ${ }^{34,35}$ por lo que en este estudio se han preferido los modelos gráficos en un abordaje meramente descriptivo.

En resumen, durante el periodo de estudio los patrones de mortalidad por accidentes de tráfico en España muestran un ligero incremento, más evidente en el grupo de edad de 15-29 años, aunque no se ajusten a una tendencia lineal. Este comportamiento de las tasas no puede ser explicado suficientemente por los efectos de la edad, el periodo de diagnóstico de la muerte y la cohorte de nacimiento. Entre las medidas de seguridad vial analizadas, vale la pena destacar el efecto protector de volver obligatorio el uso del casco y de las luces de cruce en motocicletas. 


\section{Referencias}

1. Conde V. Importancia sanitaria de los accidentes de tráfico en España. Rev Sanid Hig Publica 1989;63:19-42.

2.Altozano JM. Los accidentes de tráfico en España. En:Alcohol, drogas y accidentes de tráfico. Madrid: Ministerio de Sanidad y Consumo, 1987: 39-53.

3. Aspectos generales de los accidentes de tráfico. En:Alcohol, drogas y accidentes de tráfico. Madrid: Ministerio de Sanidad y Consumo, 1987: 13-17.

4. Prada R, Río MC del, Alvarez FJ. Accidentes de tráfico en la población española. Med Clin 1995; 105:601-604.

5. Encuesta N acional de Salud 1995. Madrid: Ministerio de Sanidad y C onsumo, 1996.

6. Dirección G eneral de Tráfico. Boletín Informativo Accidentes.Años 1970, 1975, 1980, 1985, 1990, 1995.

7. Instituto N acional de Estadística. Movimiento natural de la población española. D efunciones según la causa de muerte.Años 1970-1993, vol. III. 8. Gine JM. Mortalidad por accidentes de tráfico en Cataluña y otras comunidades autónomas (1983-1990). Gac Sanit 1992:164-169.

9. Gestal-0 tero JJ. Accidentes. En: Piedrola G. et al. Medicina preventiva y salud pública. 9a. edición. Barcelona: Masson-Salvat Medicina, 1991:955-971. 10. World Health 0 rganization. World health statistics annual. Ginebra: O MS, 1995

11. Rams MA, 0 rtega A, Sancho M. Influencia del alcohol y las drogas en los accidentes de tráfico. Rev Esp D rogodependencias 1993;18:247-252. 12. Prada R, Río MC del,Alvarez FJ. Presencia de procesos patológicos en los conductores españoles: su relevancia en el campo de la seguridad vial. Rev Esp Salud Publica 1995;69:499:508.

13. Sarasqueta $C$, Z unzunegui MV. Principales causas de mortalidad en jóvenes en la Comunidad Autónoma de Madrid (1990-1991). Gac Sanit 1994:8:117-121.

14. Perales $N$. Estrategia comunitaria ante los accidentes de tráfico.Todo Hospital 1990;63:51-64.

15. Consejo Superior de Tráfico y Seguridad de la C irculación. Plan N acional de Seguridad Vial para el año 1993. Madrid: Ministerio del InteriorDirección General de Tráfico, 1993.

16. Clasificación Internacional de Enfermedades C.I.E.-9-MC. Madrid: Ministerio de Sanidad y Consumo, 1994.

17. Instituto $\mathrm{N}$ acional de Estadística. Proyecciones de la población española para el periodo 1968-1995. Madrid: IN E, 1981.
18. Instituto N acional de Estadística. Proyecciones de la población española para el periodo 1980-2010. Madrid: IN E, 1988.

19. Segi M. Cancer mortality for selected sites in 24 countries 1950-1957. Sandai, Japón: Department of Public Health, Toboku University School of Medicine, 1960

20. C ummings SR, Hulley SB. D iseño de la investigación clínica. Barcelona: D oyma, 1993.

21.Viel JK. La régression de Poisson en épidémiologie. Rev Epidemiol Sante Publique 1994:42:79:87.

22. Holland W W, ed. Commission of the European Communities. European community atlas of avoidable death. 2 a. edición. 0 xford: 0 xford Medical Publications (series, núm.6), 1991;vol. I.

23. Malfetti JL. Drivers 55+: N eeds and problems of older drivers. Falls Church AAA: Fundation for Traffic Safety, 1985.

24. Transportation in an aging society: Improving mobility and safety for older persons.W ashington, D.C.: N ational Research Council, 1988; vols. 1 y 2.

25. Mayoral MF,Vega IL, Bueno MB,Valentín A. Los conductores mayores: dificultades en la conducción y estrategias de afrontamiento. Rev Gerontol 1993;3:86-94

26. Seguridad Vial. Prevenir por encima de todo. Muface 1997;167:24-27. 27. Martínez de Aragón MV, Llácer A. Mortalidad en España en 1992. Boletín Epidemiológico Semanal 1995;3:153-164.

28. Masa J, Pelayo A, De Irala J, Fernández-C rehuet J, Díaz C, Martínez de la Concha D. Mortalidad por accidentes de tráfico entre los 15 y 24 años: factores socioeconómicos asociados. Med Prev 1996;2:20-24.

29. Sesma FJ,A rdanaz E, Lera JM, Belzunegui T, SolaA , Gómez I. El riesgo de lesión en accidentes de tráfico por conducir bajo los efectos del alcohol en N avarra. Gac Sanit 1992;6:117-122.

30. González JC. Intervención del médico en la prevención del accidente de tráfico. Información terapéutica del SN S 1992;16;5.

31. Salleras L, Taberner JL, Fernández R, Prats R, Guayta R, Garrido $P$ et al. Consejos para la prevención de accidentes. Med Clin (Barc) 1994;102:127-131. 32. Alvarez FJ, Río MC del. Drogas, drogodependencias y seguridad vial. Rev Esp Drogodependencias 1995;19:281-285.

33. Holford TR. Understanding the effects of age, period and cohort on incidence and mortality rates. Annu Rev Public Health 1991;12:425-457. 34. D evesa SS, Blot W J, Stone BJ, Miller BA, Tazone RE, Fraumeni JF. Recent cancer trends in the United States. J N atl Cancer Inst 1995;87:175-182. 35. 0 smond C, G ardner MJ.Age, period and cohort models. N on-overlaping cohort don't resolve the identification problem. Am J Epidemiol 1989;129:31-35. 